\title{
Overhead Line Mechanics Taking the Influence of Wind into Account
}

\author{
Miroslav Müller \\ Czech Technical University in Prague, Faculty of Electrical Engineering, Department of Electrical Power Engineering, \\ Technická 2, 16627 Praha 6, Czech Republic
}

Corresponding author: miroslav.muller@fel.cvut.cz

\begin{abstract}
This paper deals with problems of overhead line motion. The line model is based on a dynamic description of a catenary curve. The benefits of dynamic modeling in this field are decrypted, and there is an explanation of one of the models that is used. The dynamic model is derived from a string equation. The main contribution of the model is in 3D simulation of complex mechanics. The model of an overhead line shaking is generally based on the superposition of harmonic components, in particular the spatial coordinates. Each individual harmonic component is solved separately in one step of calculation, and is then combined with the other solutions. The result is a continuous description of the position of the wire along its length in both the space domain and the time domain. The model thus allows calculations of uneven effects of forces along the length of an overhead line. The accuracy of the calculation is determined by the number of harmonics and other parameters that are calculated (e.g. step size, simulation time) The model is actually a combination of discrete and continuous calculations. Each model function block is described in the form of an equation. In the case study, ACSR 350/59 wire is analyzed. In this part of our work, an auxiliary model of wind influence was integrated into the global model.
\end{abstract}

Keywords: catenary, overhead line, dynamic model, 3D simulation.

\section{Introduction}

Dynamic models are advantageous when they are used for evaluating the influence of weather in dependence on position and time. Weather influences include wind, icing, increased current flow through the wire, and temperature changes. Due to their complexity, dynamic models are also useful for simulating various mechanical wave phenomena, e.g. galloping. The use of models makes analyses of characteristic oscillations more accurate and easier. The result contains stable and unstable spaces around the operating state. The system is excited in the unstable region, leading to wire damage. The model described here is a continuous3-DOF (degrees of freedom) model. Wang and Lilien [7] give a simplified modification of a 2-DOF model in a similar form.

\section{Dynamic model of wire}

The analysis of dynamic behavior is based on wave equations (1)-(3). The simulation conditions are depicted in Figures 1 and 2 - the situation can be imagined as a strained wire between two towers. The model is constrained like a string which can move between fixed points in horizontal and vertical directions, and can also rotate. The possibility of rotating a wire along its axis is important for simulations of an icy wire, and is used for solving combined tasks with icing and wind. The situation is depicted in Fig. 2.

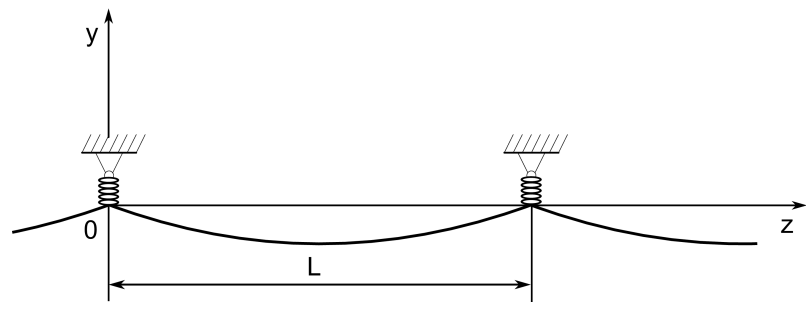

Figure 1: Overhead line field between towers.

The dynamics of the movement of a wire is described by three partial differential equations. The first equation describes the motion of the wires in the vertical direction depending on the longitudinal position (variable $z$ ) and on time $t$. The second equation describes the wire motion in the horizontal direction. The third equation then adds rotation(torsion). Each coordinate depends on the longitudinal direction and the time domain.

$$
\begin{aligned}
& m \frac{\partial^{2} y}{\partial t^{2}}+C_{y} \frac{\partial y}{\partial t}-T \frac{\partial^{2} y}{\partial z^{2}}=F_{v}(z), \\
& m \frac{\partial^{2} x}{\partial t^{2}}+C_{x} \frac{\partial x}{\partial t}-T \frac{\partial^{2} x}{\partial z^{2}}=F_{h}(z), \\
& I \frac{\partial^{2} \theta}{\partial t^{2}}+C_{\theta} \frac{\partial \theta}{\partial t}-G J \frac{\partial^{2} \theta}{\partial z^{2}}=M_{t}(z),
\end{aligned}
$$

where $m$ is the mass of the wire per $1 \mathrm{~m}$ length. Parameters $C_{y}, C_{x}$ and $C_{\theta}$ are damping coefficients, $T$ is the tension of the mechanical stress in the longitudinal direction of the wire, $I$ is the moment of inertia, $G_{J}$ is the torsional stress. The right side of the equa- 


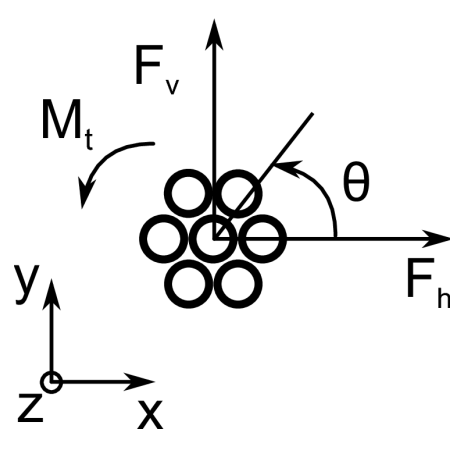

Figure 2: Forces and moment of the force affecting the wire.

tion describes the source of exciting forces, and the torque forces. Constant $L$ is the total length of the wire.

The solution of the system of equations depends strongly on the boundary conditions. In this case, the wires are fixed at both ends, and thus:

$$
\begin{gathered}
x(z=0, t)=y(z=0, t)=\theta(z=0, t)=0, \\
x(z=L, t)=y(z=L, t)=\theta(z=L, t)=0 .
\end{gathered}
$$

The solving process for a system of wave equations (1)-(3) is based on the possibility of separating the variables (position and time). The variables on right side are expressed by means of Fourier transformation. This results in a new set of equations for each harmonic:

$$
\begin{aligned}
& m \frac{\mathrm{d}^{2} x_{k}}{\mathrm{~d} t^{2}}+C_{x} \frac{\mathrm{d} x_{k}}{\mathrm{~d} t}+T\left(\frac{k \pi}{t}\right)^{2} x_{k} \\
& =\frac{2}{L} \int_{0}^{L} F_{v}(z) \cdot \sin \frac{k \pi z}{L} \mathrm{~d} z, \\
& m \frac{\mathrm{d}^{2} y_{k}}{\mathrm{~d} t^{2}}+C_{y} \frac{\mathrm{d} y_{k}}{\mathrm{~d} t}+T\left(\frac{k \pi}{t}\right)^{2} y_{k} \\
& =\frac{2}{L} \int_{0}^{L} F_{h}(z) \cdot \sin \frac{k \pi z}{L} \mathrm{~d} z, \\
& I \frac{\mathrm{d}^{2} \theta_{k}}{\mathrm{~d} t^{2}}+C_{\theta} \frac{\mathrm{d} y_{k}}{\mathrm{~d} t}+G J\left(\frac{k \pi}{t}\right)^{2} \theta_{k} \\
& =\frac{2}{L} \int_{0}^{L} M_{t}(z) \cdot \sin \frac{k \pi z}{L} \mathrm{~d} z .
\end{aligned}
$$

The resulting position of the wire is obtained by superposingthe solutions of (4)-(6) for each calculated harmonic.

\subsection{Excitation forces}

The model of the excitation forces takes into account the gravitational forces and the forces induced by wind motion and the consequent drift of the wire.

The gravitational force is a simulated as a uniform load, and the force induced by the gravitational field effects is given by:

$$
F_{v}=-m g,
$$

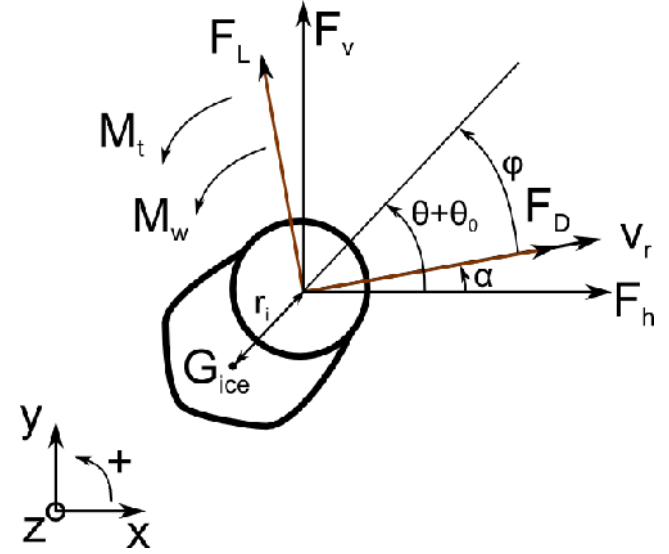

Figure 3: Wind influence — diagram showing angles and forces.

where $g$ is the gravitational constant and $m$ is the weight of the conductor per meter, including ice.

The effect of wind is given by the wind resistance of the wire. In this case, the wire is shifted and rotated. The constants describing the relationship between the wire and the air are:

$$
\begin{aligned}
k_{D} & =\frac{1}{2} \rho_{a i r} \phi_{c o n d} \\
k_{M} & =\frac{1}{2} \rho_{a i r} \phi_{c o n d}^{2}
\end{aligned}
$$

where $\rho_{\text {air }}$ is air density, and $\phi_{\text {cond }}$ is wire diameter. Wind acting on the wire results in two orthogonal components of the forces. The first of these is force , applied in the direction of the wind motion and thus the main direction. The second force is the orthogonal component, which is orthogonal to the main direction of the wind. The wire is also influenced by torque force, which rotates both the wire and the ice. The position and orientation, including the action of the forces, is shown in Fig. 3. The ice element is also marked in the figure. These component forces are described by the set of equations (7)-(9).

$$
\begin{aligned}
F_{D} & =v_{r}^{2} k_{D} C_{D}(\phi), \\
F_{L} & =v_{r}^{2} k_{D} C_{L}(\phi), \\
M_{\omega} & =v_{r}^{2} k_{M} C_{M}(\phi) .
\end{aligned}
$$

Parameter $v_{r}$ is relative wind speed, $C_{D}, C_{L}$ and $C_{M}$ are the transformation function describing the distribution of the forces and torque components depending on directional angle $\phi$. These functions have to be obtained from measurements in an aerodynamic tunnel.

Relative wind speed is another problem to be solved. The speed is calculated as a relative value, because the wire generally moves too. The velocity 
components are as follows:

$$
\begin{aligned}
& v_{r x}=v_{0 x}-\frac{\partial x}{\partial t}-r_{i} \frac{\partial \theta}{\partial t} \sin \left(\theta+\theta_{0}\right) \\
& v_{r y}=v_{0 y}-\frac{\partial y}{\partial t}-r_{i} \frac{\partial \theta}{\partial t} \cos \left(\theta+\theta_{0}\right)
\end{aligned}
$$

where, $v_{0 x}, v_{0 y}$ are the components of wind velocity, $x$ and $y$ are the position of the conductor, $r_{i}$ is the distance between center of gravity of the conductor and the center of gravity of the ice, $\theta$ is the rotation of the wire to rest position $\theta_{0}$,

$$
v_{r}=\sqrt{v_{r x}^{2}+v_{r y}^{2}}
$$

Angle $\phi$ is a necessary component for functions $C_{D}$, $C_{L}, C_{M}$. The value is determined by:

$$
\begin{aligned}
\tan \alpha & =\frac{v_{r x}}{v_{r y}}, \\
\varphi & =\theta+\theta_{0}-\alpha-\frac{r_{i}}{v_{0}} \frac{\partial \theta}{\partial t} .
\end{aligned}
$$

Since forces $F_{d}$ and $F_{L}$ are described in the system with the wind direction, and the other equations are in a stationary system in ground coordinates, it is necessary to transform the result into coordinates $F_{v}$ and $F_{h}$ :

$$
\begin{aligned}
& F_{v}=F_{L} \cos \alpha+F_{D} \sin \alpha-m g \\
& F_{h}=-F_{L} \sin \alpha+F_{D} \cos \alpha
\end{aligned}
$$

\section{Case study}

The simulations are based on the equations described in the previous section. ACSR 350/59 wire was chosen for the case study simulation. Functions $C_{D}$, $C_{L}$ and $C_{M}$ were taken from the literature [4], because complex technical measuring equipment would be needed to measure them (an aerodynamic tunnel with a tight wire and a controlled climatic environment). The span length was set as $a=244 \mathrm{~m}$ and $h \approx 0 \mathrm{~m}$ (difference in height between the conductor support points). The icing of the wire was $0.6 \mathrm{~kg} / \mathrm{m}$, and the tension was assumed to be $T=35600 \mathrm{~N}$.

\subsection{Basic simulation}

The simulation shows the step response of the new wire under constant wind speedinfluence $v_{0}=$ $(16,5.2) \mathrm{m} / \mathrm{s}$ condition (the relative angle position of the icing is assumed to be steady). Only odd harmonics were taken account (in the range 1st to 7 th) in the calculation. The even harmonics are zero functions in this case.

Figures 4-6 show the wire positions for $z=L / 2$ (the middle of the wire span) in time. Since the wire is influenced by a constant wind flow $v_{0}$, and the

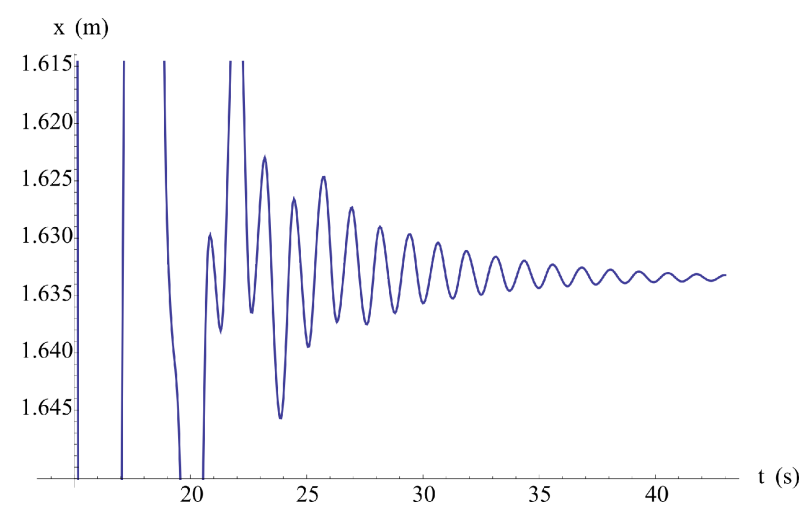

Figure 4: $x$-coordinates in the middle of the wire $\operatorname{span}(z=L / 2)$.

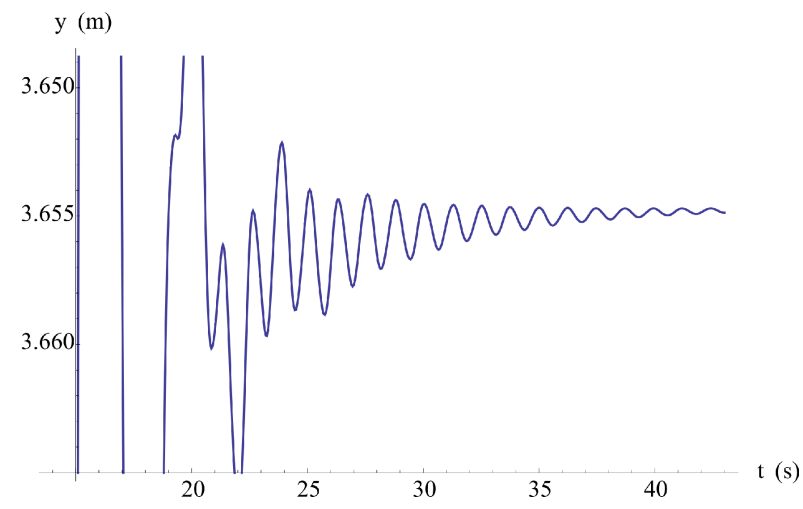

Figure 5: $y$-coordinates in the middle of the wire $\operatorname{span}(z=L / 2)$.

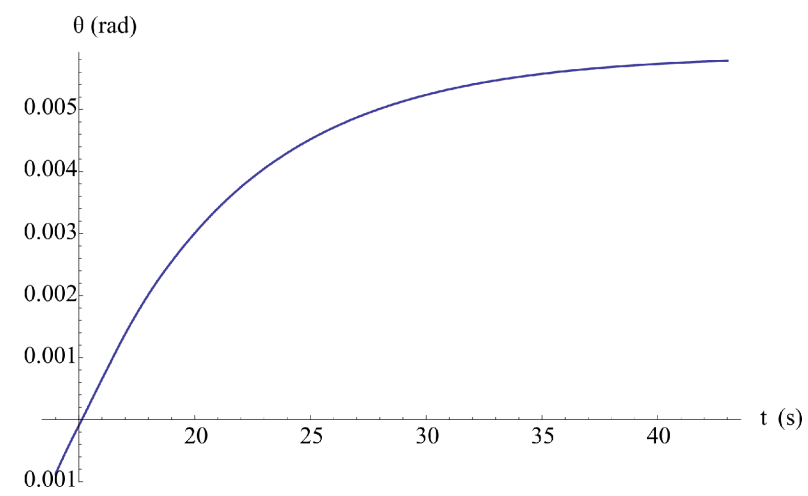

Figure 6: Rotation of the wire $\theta$ in the middle of the wire $\operatorname{span}(z=L / 2)$. 


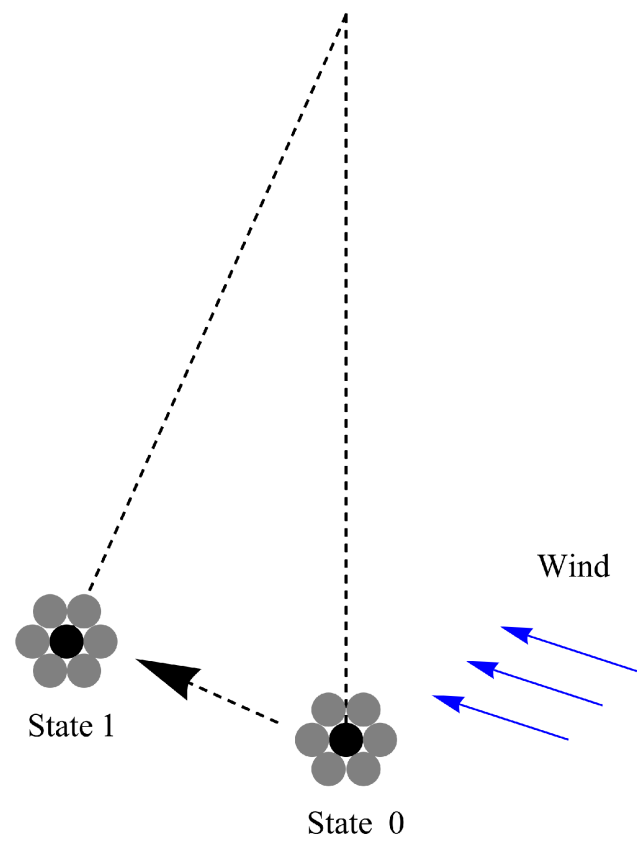

Figure 7: Schematic representation of the movement of the wire due to the influence of wind.

system is in a stable state, the position of the wire results in a stable equilibrium. The situation is shown schematically in Figure 6, where "State 0" is steady equilibrium for the state without wind and "State 1" is a stable position with the influence of a constant wind $v_{0}$. Coordinates y contain gravitational forces causing an offset.

To determine the position of each coordinate, it is necessary to evaluate some harmonics (4)-(6) for each component. Sub-harmonics results are shown in Figures 8 to 10. Since the own load of the conductor is constant, and other parameters are also homogeneous (including icing), the solutions do not contain even harmonics. This simplifies the calculation, and even harmonics do not have to be taken into account. In the case of a non-constant load, this simplifying assumption does not generally apply.

\subsection{Effect of icing position}

The following simulation is done under the same conditions as above. The only change is in the variation of the icing position angle (see Fig. 3). Steady state results are presented in Tab. 1. These values show that icing formed under windy conditions leads to results that are far from homogeneous.

\subsection{Influence of harmonics calculated on the basis of simulation results}

The influence of harmonic components, taking into account the calculated steady state, is shown in Table 2. Final number of harmonics used in the simulation

\begin{tabular}{rccc}
\hline$\theta_{0}$ & $\begin{array}{c}x \\
(z=L / 2) \\
\left(\text { in }^{\circ}\right)\end{array}$ & $\begin{array}{c}y=L / 2) \\
(\text { in } \mathrm{m})\end{array}$ & $\begin{array}{c}\theta=L / 2) \\
(\text { in } \mathrm{m})\end{array}$ \\
\hline 18 & -1.199 & -3.819 & 0.007 \\
\hline 0 & -1.355 & -3.891 & 22.074 \\
\hline-18 & -1.619 & -3.677 & 20.375 \\
\hline-36 & -1.877 & -3.250 & -15.042 \\
\hline-54 & -2.079 & -2.895 & -78.379 \\
\hline
\end{tabular}

Table 1: Steady state values for various angle.

\begin{tabular}{cccc}
\hline $\begin{array}{c}\text { Harmonic } \\
\text { order }\end{array}$ & $\begin{array}{c}x=L / 2) \\
(\text { in m })\end{array}$ & $\begin{array}{c}y=L / 2) \\
(\text { in m })\end{array}$ & $\begin{array}{c}(z=L / 2) \\
\left(\text { in }^{\circ}\right)\end{array}$ \\
\hline 1 & -1.68764 & -3.77506 & 0.00607 \\
\hline 3 & -1.62494 & -3.63551 & 0.00585 \\
\hline 5 & -1.63849 & -3.66566 & 0.00589 \\
\hline 7 & -1.63355 & -3.65467 & 0.00588 \\
\hline 9 & -1.63587 & -3.65984 & 0.00588 \\
\hline
\end{tabular}

Table 2: Steady state value for various harmonics taken into account.

is determined by testing the convergence of the result. It is not sufficient only to decompose the resulting values in the steady state. A simple decomposition cannot be used because the system is nonlinear.

The values show that the effect of the harmonics on the results decreases as the order of the harmonics rises. The effect of 9th and higher harmonics is therefore low, and their added value is negligible in comparison with the complexity of the calculation.

\section{Conclusion}

This paper describes a 3-DOF model for simulating the dynamic behavior of a stretched wire. The main external influences on the model have been described. The effect of wind on a conductor with icing has also been described.

A case study has shown a wire with constant icing and steady wind. In the case study, the simulations show the position of the middle of the span for all 3 coordinates. In addition to that, the behavior of each harmonic is calculated. The courses of displacements $x, y, \theta$ depending on coordinate $\mathrm{z}$ can be viewed for other locations, but it has no real application. This part of our work also investigated what 

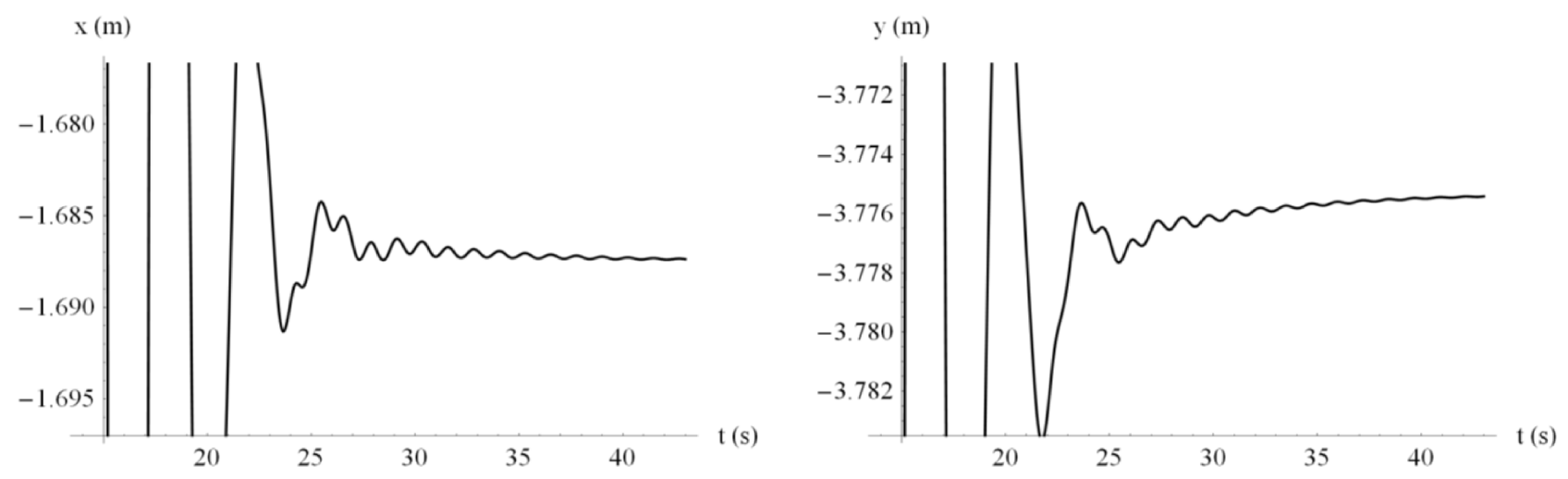

Figure 8: The 1st harmonic amplitudes of a position in time.
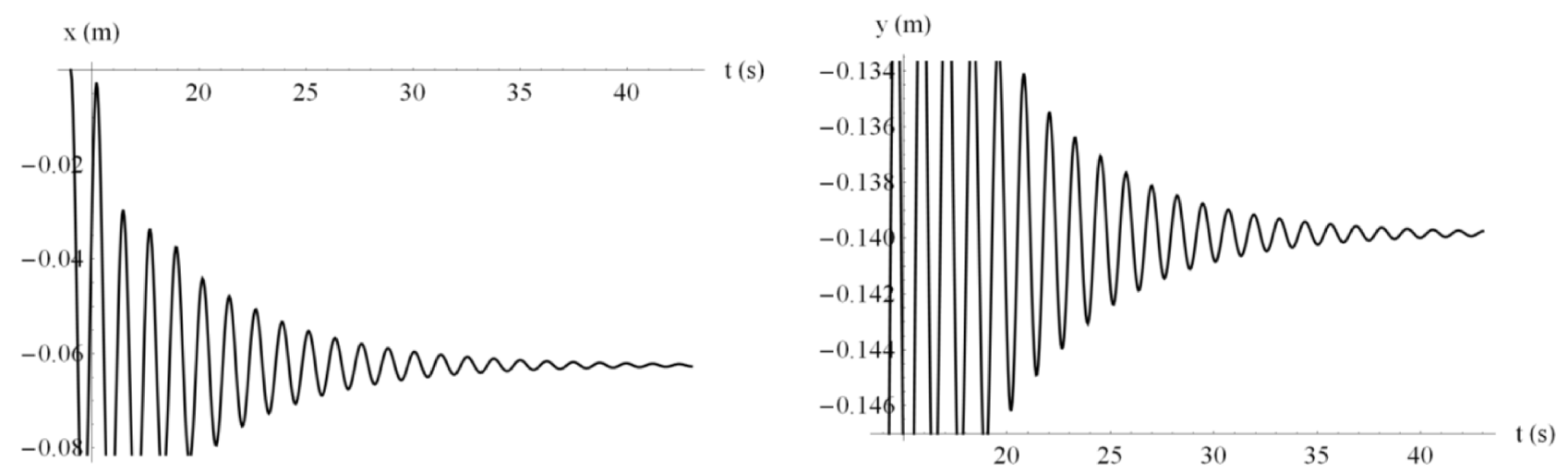

Figure 9: The 3rd harmonic amplitudes of a position in time.
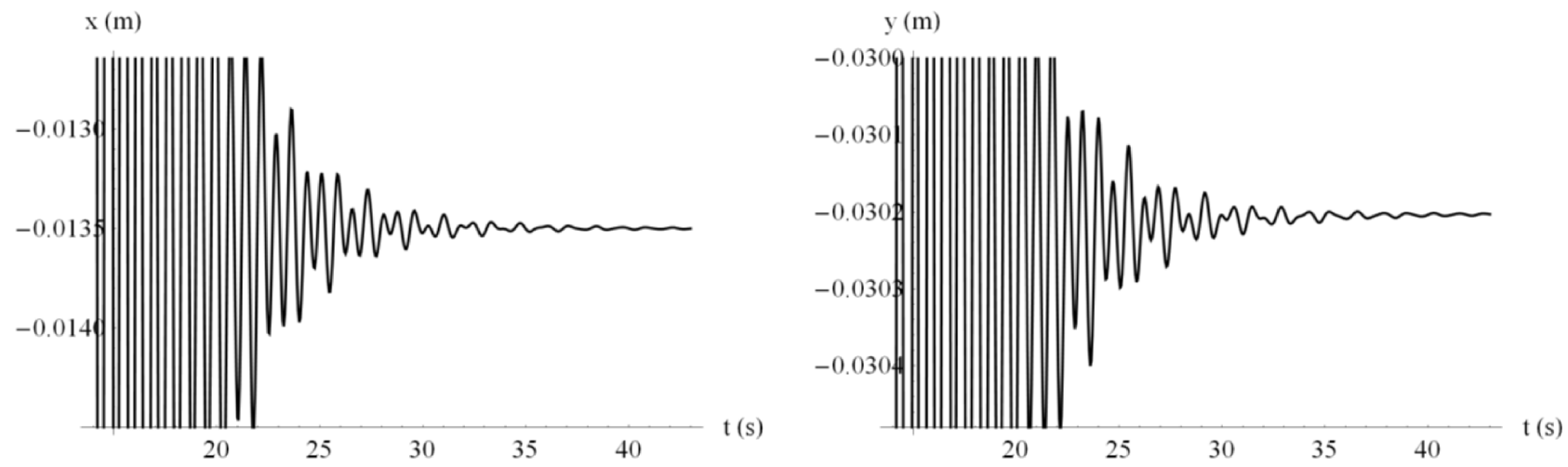

Figure 10: The 5th harmonic amplitudes of a position in time. 
happens when a frozen wire turns. The simulation shows that a wire with significant icing placed in one direction (a shift of the center of gravity from the center of the wire) is very sensitive to the wind parameters (Tab. 1). In real situations, icing is placed asymmetrically. In this case, it is possible to choose a characteristic direction and use an average value. For a more accurate result, it would be necessary to describe the distribution function for the icing, including the directional angle and the shift of gravity along the wire length $z$.

The study also calculated the influence of the number of equations that was solved, where a set of 3 equations represents one harmonic. The simulation and the model results show that the even harmonics do not affect the solution for uniform distribution of forces and weight. In addition, the influence of the 9th and high order harmonics is negligible for the simulation in comparison with the increasing complexity of the calculation.

\section{Acknowledgements}

Financial support from the Ministry of Education, Youth and Sports, through grant number MSM 6840770017 , is gratefully acknowledged.

\section{References}

[1] R. Claren, G. Diana. Mathematical Analysis of Transmission Line Vibration. IEEE Transactions on Power Apparatus and Systems Pas88(12):1741-1771, 1969.
[2] F. Kiessling, P. Nefzger et al. Overhead Power Lines - Planning, Design, Construction, Springer, Germany, 2003.

[3] Lanové vodice. ŽHS, Žiar nad Hronom, 2007, http://www.lana.sk, [2012-08-01].

[4] J. L. Lilien. Overhead Line Vertical Galloping on Bundle Configurations: Stability Criterions and Amplitude Prediction. In Overhead Line Design and Construction: Theory and Practice, London, 1989, pp. 65-69.

[5] J. L. Lilien, J. Wang, J. et al. Overhead Transmission Lines Design: Some Mechanical Aspects. In ICPST'94, Beijing, 1994.

[6] C. B. Rawlins. An Efficient Method for Measuring Dissipation by Dampers in Laboratory Spans. IEEE Transactions on Power Delivery 3(3):1146-1156, 1988.

[7] J. Wang, J. L. Lilien. Overhead Transmission Line Galloping. A Comparative Study between 2-DOF and 3-DOF Models. In 3ème Congrès National Belge de Mécanique Théorique et Appliquée, Liège, 1994, pp. 257-261.

[8] J. Wang, J. L. Lilien. Overhead Electrical Transmission Line Galloping - A Full Multi-Span 3DOF Model, Some Applications and Design Recommendations. IEEE Transactions on Power Delivery 13(3):909-916, 1998. 